\title{
Measurement of burnout: Validation of the Sinhala translation of Maslach Burnout Inventory - Educators Survey among female primary school teachers in Sri Lanka
}

\author{
De Silva PV ${ }^{1}$, Hewage $C^{2}$, Fonseka $P^{1}$ \\ ${ }^{1}$ Department of Community Medicine, ${ }^{2}$ Department of Psychiatry, Faculty of Medicine, University of Ruhuna, \\ Galle, Sri Lanka
}

Correspondence: Dr.P.V.de Silva (pvijithadesilva123@yahoo.com)

\begin{abstract}
Background and Objective: Burnout was recognized as a disease entity about three decades ago and was found to be highly prevalent among the human service professionals. Objective of this study was to translate the Maslach Burnout Inventory- Educators Survey (MBI-ES) in to Sinhala and to validate it for the identification of burnout among female teachers of Sri Lanka.

Methods: Translation was carried out using combined qualitative and quantitative methods with a nominal group. Face, content and consensual validity were assessed by a group of experts. To determine the criterion validity, total scores of MBI-ES-Sinhala version (MBI-ES-Sin) were compared with a diagnosis made by a consultant psychiatrist. Using a Receiver-operated Characteristic (ROC) Curve, suitable cut-off points were determined. Test- retest reliability was measured using paired t-test. Cronbach's alpha was used to measure the internal consistency.

Results: Face, content, consensual and criterion validity of MBI-ES-Sin were found to be satisfactory. Total MBI-ES-Sin score 54 was taken as the cut-off point with a sensitivity of $92 \%$ and specificity of $90.4 \%$. Testretest reliability was found to be satisfactory. The internal consistency in each sub-scale and also in the total scale was satisfactory.

Conclusion: MBI-ES-Sin is a reliable and a valid instrument to measure burnout among female teachers in Sri Lanka. Therefore, we can further conclude that the methods used in the translation and validation in this study are appropriate and can be used to translate and validate MBI-ES to any other language.
\end{abstract}

Keywords: Burnout, Translation, Validation, Maslach Burnout Inventory

\section{Introduction}

Stress and stress-related problems, such as burnout are becoming increasingly common among working populations throughout the world (1). Nowadays mental health problems contribute to a significant proportion of occupational health problems and it is one of the commonest causes for ill health, retirement, sickness and absence. Therefore, large numbers of scientists in the developed world are engaged in research and preventive work on job related mental health problems (2).
About three decades ago, a new disease entity called burnout was identified. Different researchers have defined burnout differently. However, the most commonly accepted definition came from Maslach in 1981. She defines burnout as emotional exhaustion, depersonalization and reduced personnel accomplishment that can occur among individuals who do "People work of some kind" (3). She further describes burnout as a psychological process that begins when human service professionals are overwhelmed with the unexpected 
and unbearable stressful aspects of the job that frustrate their efforts to make a positive impact on others. As described in the definition of burnout, it has been shown to be most prevalent among human service professionals such as nurses, teachers, social workers and mental health workers $(4,5)$.

Most studies on burnout have been done in the developed world where the socio-cultural status is significantly different to that in countries in the South Asian region including Sri Lanka.

Teaching is one of the most important occupations involved with many stressful events. During the last few years, there were numerous reports of stress and stress-related problems among teachers and they were attributed to the recent curricular changes, more demands for student achievements and parental pressure (6). Some of the research also found, teaching as a high stress profession (7).

We were unable to find studies related to burnout in Sri Lanka. The paucity of studies probably reflects the lack of a suitable tool in local languages to measure burnout among susceptible groups in Sri Lanka. With the recent rapid socio-economic changes in the country the need of a reliable tool to measure burnout is strongly felt. This study was planned to translate and validate an instrument to measure burnout among school teachers in Sri Lanka.

Maslach Burnout Inventory - Educators survey (MBI-ES) was selected for this purpose since it has been widely used in different parts of the world in different languages and found to have satisfactory level of validity. More than $90 \%$ of journal articles on burnout are based on the MBI (8). It has 22 items in three subscales namely emotional exhaustion, depersonalization and reduced personnel accomplishment. All the items have to be given a score in a Likert scale ranging from 0 (never) to 6 (every day). High values in emotional exhaustion and depersonalization and low values of personal accomplishment indicate burnout. Its ability to use as a self-administered questionnaire is an added advantage.

\section{Methods}

Translation of the original English questionnaire to Sinhala language was carried out using a combined qualitative and quantitative method with a nominal group. This group $(n=6)$ consisted of a community physician, a psychiatrists (2), postgraduate trainees (2) and a female school principal. Before the translation process, the group assessed the conceptual equivalence of the MBI-ES to the Sri Lankan culture. During the translation the experts assessed the item, semantic and operational equivalences. Consensus methods were used to determine the extent to which members of a nominal group agreed about a given issue (consensus measurement) and to resolve disagreement (consensus development) (9).

The MBI-ES-Sinhala version (MBI-ES-Sin) was pre-tested among 25 female primary school teachers. The objective of this pre-test was to find out whether this questionnaire was easily understandable and to determine the time necessary to complete it. It took about 10 minutes to complete it. After the pre-test a few minor modifications were carried out according to the findings.

In the present study, the translated MBI-ES-Sin was subjected to judgmental validity (face, content and consensual validity) and criterion validity. Judgmental validity was assessed by a group of experts including community physicians (2), a psychiatrist, and an experienced teacher. Group members appraised the relevance of the translated tool to the three domains of burnout and assessed the face validity. The panel assessed the content validity by checking whether all the aspects of the original MBI-ES were covered by the MBI-ES-Sin. Consensual validity was determined by assessing the agreement of the panel members regarding the appropriateness of the translated conceptual definition of burnout and operational terms in the tool.

Criterion validity involves comparisons of results obtained from the tool with a gold standard. In the absence of any objective gold standard for a psychiatric case, an interview by a psychiatrist will become the ultimate diagnosis. There was no gold standard to measure burnout in the community. Therefore, findings based on the questionnaire were compared with the diagnosis made by an experienced consultant psychiatrist. Depending on the expected specificity and the sensitivity of the MBI-ES-Sin, the minimum sample size required for the validation was calculated as 49 teachers with burnout and 49 teachers without burnout. Teachers 
were selected from one educational division in the western province of Sri Lanka, using a nonprobability convenient sampling method. Only Sinhala medium female primary school teachers were selected for the study. Teachers who had teaching experience less than six months were excluded.

Administration of the MBI-ES-Sin was carried out by the principal investigator. Each teacher was then directed to the consultant psychiatrist, who conducted a clinical interview and made the clinical diagnosis. The consultant psychiatrist carried out this procedure till the required number of subjects was selected. All the assessments were done by the same consultant psychiatrist to eliminate interobserver variability.

A composite total score of burnout was constructed by summing the items in MBI-ES-Sin, after reverse keying those of personal accomplishment. These scores and the clinical diagnosis were used to graph a receiver operator characteristics (ROC) curve and by that obtained the appropriate cut off levels for the total MBI-ES-Sin score. Test- retest reliability was measured using paired $t$ test. For this purpose MBIES-Sin was re-administered to 25 teachers after one week. Cronbach's alpha was used to measure the internal consistency. Same data used for the validation study was used for this purpose also.

\section{Results}

Panel of experts had rated all the items in the MBIES-Sin as appropriate to measure burnout among female primary school teachers in Sri Lanka. Fifty teachers with burnout and 73 teachers without burnout were assessed using both MBI-ES-Sin and clinical diagnosis to measure the criterion validity. Using those data, a ROC curve was drawn by plotting the sensitivity against 1- specificity using the SPSS software package. Area under the curve was 0.944 with a $95 \%$ confidence interval of $0.887-0.977$. The point where the curve turns to the right was taken as the best cut off point. In this situation, specificity was also considered, to minimize the false positives. According to the coordinates of the curve, the best cut off point to identify cases of burnout and noncases was considered to be 54 with the sensitivity of $92 \%$ and specificity of $90.41 \%$ (Table 1 ). The positive predictive value was $86.79 \%$ and the negative predictive value was $94.28 \%$.
Table 1: Coordinates of the curve

\begin{tabular}{ccc}
\hline $\begin{array}{c}\text { Positive if greater } \\
\text { than or equal }\end{array}$ & Sensitivity & Specificity \\
\hline 50 & 98.00 & 72.6 \\
51 & 98.00 & 74.0 \\
52 & 98.00 & 76.7 \\
53 & 96.00 & 84.9 \\
$\mathbf{5 4 *}$ & $\mathbf{9 2 . 0 0}$ & $\mathbf{9 0 . 4}$ \\
55 & 88.00 & 91.8 \\
56 & 82.00 & 93.2 \\
57 & 66.00 & 94.5 \\
58 & 56.00 & 94.5 \\
59 & 46.00 & 95.9 \\
60 & 42.00 & 95.9 \\
\hline
\end{tabular}

To measure the test retest reliability, two sets of data were compared using the paired t-test. According to the test, the difference of 25 pairs was not significant at 0.05 level. To measure the internal consistency, Cronbach's alpha was calculated for the three subscales of the MBI-ES-Sin and for the total MBIES-Sin scores (Table 2).

Table 2: Internal consistency of the MBI-ES-Sin and it's sub scales

\begin{tabular}{|l|c|}
\hline \multicolumn{1}{|c|}{ Scale } & $\begin{array}{c}\text { Cronbach's } \\
\text { Alpha }\end{array}$ \\
\hline Emotional Exhaustion & 0.90 \\
\hline $\begin{array}{l}\text { Reduced personal } \\
\text { accomplishment }\end{array}$ & 0.78 \\
\hline Depersonalization & 0.82 \\
\hline Total MBI-ES score & 0.88 \\
\hline
\end{tabular}

\section{Discussion}

In the present study MBI-ES was translated to Sinhala language according to the combined qualitative and quantitative methods using a nominal group. The group members taking into consideration the cultural issues and the level of education among female primary school teachers assessed every item of the questionnaire individually. This method of translation was used in preference to translation and 
back translation method. In the translation and back translation method, translators try to translate the instrument taking all measures to minimize the change between translated and original version. Some scientists describe this method as a technical way of translation and stressed the need of more rigorous systematic and contextual approach to translation (10). On the other hand the combined qualitative and quantitative method, which was used in the present study, mainly preserves the conceptual domains of the original questionnaire rather than the structure of any particular language.

In the present study Area Under the Curve is 0.94 and it is very close to 1 . It confirms that MBI-ES-Sin questionnaire has good ability to identify teachers with and without burnout. Cut-off point for burnout among female teachers was determined as 54 . Since burnout is associated with possible stigma in the society, the cut-off point was selected considering both sensitivity and specificity. A sensitivity of $92 \%$ and specificity of $90.4 \%$ was obtained with the above cut-off value and therefore, it will yield minimum number of both false positives and false negatives. However, no attempts were made to validate the three subscales individually due to practical consequences and therefore, no individual cut-offs were identified.

All the values for the Cronbach's alpha for three subscales and to the total MBI-ES-Sin score were above 0.6 and it confirmed the good internal consistency in MBI-ES-Sin. The highest internal consistency was found in the emotional exhaustion subscale. Similar results were found by Iwanicki and Schwab among Massachusetts's teachers and by Gold among California teachers $(11,12)$. Buunk et al summed up all the items of the MBI and calculated the Cronbach's alpha to the total MBI score, and it was found to be 0.87 (13). This value is very close to the value, 0.88 that is obtained in the present study. Test- retest reliability confirmed the reliability of the instrument.

Considering the overall results of the present study we can conclude that MBI-ES-Sin as a valid and reliable instrument to measure burnout among female teachers in Sri Lanka. Therefore, we can further conclude that the methods used in the translation and validation in this study are appropriate and can be used to translate and validate MBI-ES to any other language.

\section{References}

1. NIOSH. Stress at work U.S. National Institute for Occupational Safety and Health, DHHS (NIOSH), 1999: Publication Number 99-101.

2. Glozier N. Mental ill health and fitness for work. Journal of Occupational and Environmental Medicine 2002; 59: 714-20.

3. Maslach C. Measurement of experience burnout. Journal of Occupational Behaviour 1981; 2: 99-113.

4. Pick D, Leiter MP. Nurses perceptions of the nature and causes of burnout. Canadian Journal of Nursing Research 1991; 23: 33-48.

5. Demirel Y, Guler N, Thoktamis A, Ozdemir D, Sezar RE. Burnout among high school teachers in Turkey. Middle East Journal of Family Medicine 2005; 3: 3.

6. Jevne R, Zingle H. Living with broken dreams; a study of teachers' health in Alberta: Alberta school employee benefit plan 1991.

7. Kyriacou C, Chien P. Teachers stress in Taiwanese primary schools. Journal of Educational Enquiry 2004; 5: 2-5.

8. Shaufeli WB, Baker AB, Hoogduin K, Schaap CAS, Kladlera A. On the clinical validity of the maslach burnout inventory and the burnout measure. Psychology and Health 2001; 16: 565-82.

9. Sumathipala A, Murray J. New approach to translate instruments for cross cultural research: a combined qualitative and quantitative approach for translation and consensus generation. International Journal of Methods in Psychiatric Research 1998; 9: 87-95.

10. Kleinman AA. Anthropology and Psychiatry: The role of culture in cross cultural research on illness. British Journal of Psychiatry. 1987; 154: 447-54.

11. Iwanicki EF, Schwab RL. A cross-validational study of the Maslach Burnout Inventory. Educational and Psychological Measurement 1981; 41: 1167-74.

12. Gold Y. The relationship of six personal and life history variables to standing on three dimensions of the Maslach burnout Inventory in a sample of elementary and junior high school teachers. Educational and Psychological Measurement 1985; 45: 377-87.

13. Buunk BP, Ybema JF, Gibbons FX. The affective consequences of social comparison as related to professional burnout and social comparison orientation. European Journal of Social Psychology 2001; 31: 337-51. 